\title{
Automated spherical aberration correction in scanning confocal microscopy
}

\author{
H. W. Yoo, ${ }^{1,2, a)}$ M. E. van Royen, ${ }^{3}$ W. A. van Cappellen, ${ }^{3}$ A. B. Houtsmuller, ${ }^{3}$ \\ M. Verhaegen, ${ }^{1}$ and $\mathrm{G}$. Schitter ${ }^{2}$ \\ ${ }^{1}$ Delft Center for Systems and Control, Delft University of Technology, Mekelweg 2, \\ 2628 CD Delft, The Netherlands \\ ${ }^{2}$ Automation and Control Institute, Vienna University of Technology, Gusshausstr. 27-29, 1040 Vienna, Austria \\ ${ }^{3}$ Erasmus Optical Imaging Center, Department of Pathology, Josephine Nefkens Institute, \\ Erasmus Medical Center, Dr. Molewaterplein 50, 3015 GE, Rotterdam, The Netherlands
}

(Received 15 September 2014; accepted 1 December 2014; published online 18 December 2014)

\begin{abstract}
Mismatch between the refractive indexes of immersion media and glass coverslips introduces spherical aberrations in microscopes especially for high numerical aperture objectives. This contribution demonstrates an automated adjustment of the coverslip correction collar in scanning confocal microscopy to compensate for spherical aberrations due to coverslip thickness mismatch. With a motorized coverslip correction collar, the adjustment procedure consists of $x z$ image scans, image processing, correction quality evaluation, the mismatch estimation, and eventually the optimal adjustment of the correction collar. For fast correction with less photodamage, coarse-fine Gaussian fitting algorithms are proposed and evaluated with various specimen for their estimation accuracy. The benefits of the proposed automated correction are demonstrated for various coverslips with biological specimens, showing the optimized resolution of the confocal microscope. (C) 2014 AIP Publishing LLC. [http://dx.doi.org/10.1063/1.4904370]
\end{abstract}

\section{INTRODUCTION}

Confocal microscopy and fluorescent labeling technologies have gone through impressive progress in the past decades and have provided powerful instruments for biomedical research. ${ }^{1-7}$ The optimal resolution of confocal microscope systems is theoretically limited by the diffraction of the light. In a practical setting, however, the loss of performance compared to the theoretical values is largely due to the mismatch between the refractive index of the high-numericalaperture objective lenses of the microscope and the refractive index of the specimen.

One common aberration occurring in optical microscopy is spherical aberration, mainly introduced by layered structures. ${ }^{8-10}$ Fig. 1 shows a spherical aberration generated by focusing through two different media. With a water immersion objective, for example, the refraction index mismatch between the immersion medium (water) and the coverslip (glass) generates spherical aberrations. Perfect compensation is difficult to achieve by a static objective design since the level of spherical aberrations is mainly fluctuated by the thickness variation of the coverslip. To cope with the varying spherical aberrations induced by coverslip thickness mismatch, microscope manufacturers provide an objective lens with a coverslip correction collar that allows adjustment of a lens block in the objective, ${ }^{11}$ which can be motorized. ${ }^{12} \mathrm{Mi}-$ croscope manufacturers recommend users to maximize the intensity of the interface reflection between the coverslip and specimen to find the best adjustment. ${ }^{8}$ Some commercial systems utilize additional sensors that measure the coverslip thickness $^{13}$ or the spherical spherical aberrations directly ${ }^{14}$

a)Electronic mail: yoo@acin.tuwien.ac.at to adjust the correction collar. Another approach adds a relay optics between the objective and tube lens for correcting spherical aberrations for deep specimen imaging. ${ }^{15}$ However, the sensors do not use the same optical path of microscope imaging, and the auxiliary sensors and devices add complexity to the microscope system. A correction based on image quality measures of these reflection is defined and evaluated without biological specimen by maximizing image sharpness find the optimal adjustment by Gaussian fitting (GF). ${ }^{16}$ However, the manual adjustment is difficult, imprecise and time consuming, causing photodamage to the specimen. Moreover, the spatial fluctuation of reflection by specimen makes it difficult for users to find the best correction.

In this paper, an automated correction of spherical aberrations is proposed based on a motor driven correction collar ${ }^{17}$ in combination with algorithms on a generalized correction quality measure. The paper is organized as follows: Sec. II describes the setup with a new motorized correction collar. In Sec. III, an axial image model is derived to design a noise reduction filter and to analyze the residual spherical aberration. A sequence of normalized axial images is recorded and the optimal correction collar adjustment is determined by the correction quality measures and correction methods presented in Sec. IV. Section V demonstrates the performance of the correction methods and improved image resolution of fluorescent specimen by experimental results obtained by the automatically adjusted confocal microscope.

\section{SYSTEM DESCRIPTION}

The overall structure of the automatic coverslip thickness mismatch correction system is illustrated in Fig. 2. An excitation laser is focused by an objective lens (HCX PL $\lambda_{B L}$ 


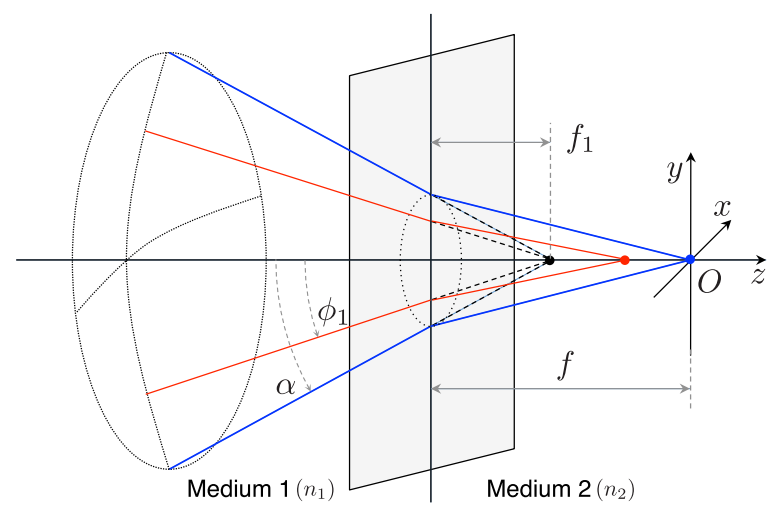

FIG. 1. Geometrical optics interpretation of the spherical aberrations caused by focusing a beam through two media $\left(n_{2}>n_{1}\right)$. The designed focal point of the lens in medium 1 (black dot) is diverged into two foci (red and blue dots), leading to image degradation.

APO $63 \times 1.20$ NA water immersion, Leica Microsystems, Mannheim, Germany). The reflections between the coverslip and specimen are collected by the same objective lens, and detected by the photomultiplier tube (PMT) with a wavelength window between $485 \mu \mathrm{m}$ and $491 \mu \mathrm{m}$ in the confocal scanhead (TCS SP5, Leica Microsystems). For obtaining axial scans, the specimen slide is linearly translated by a galvanometer-driven $z$-stage (Super Z-Galvo Stage, Leica Microsystems). The axial images are recorded by a computer, which also controls the microscope. To actuate the coverslip correction collar, a stepper motor (15HS-012, Mclennan Servo Supplies Ltd., Surrey, UK) driven by a pulse generator (Stellaris Stepper Motor Reference Design Kit, Texas Instruments, Dallas, TX, USA) adjusts the angle of the coverslip correction collar $r$ through a timing belt with a gear ratio of $1 / 6$, providing a resolution of $0.33^{\circ} /$ step over the full rotational range of $115^{\circ}$ of the correction collar.

\section{AXIAL IMAGE MODEL OF THE COVERSLIP MISMATCH PROBLEM}

\section{A. Axial image model of the reflective planar interface}

Imaging with coverslip mismatch can be modeled as imaging through two different media as shown in Fig. 1, where $n_{1}$ is the reflective index of the immersion media, and

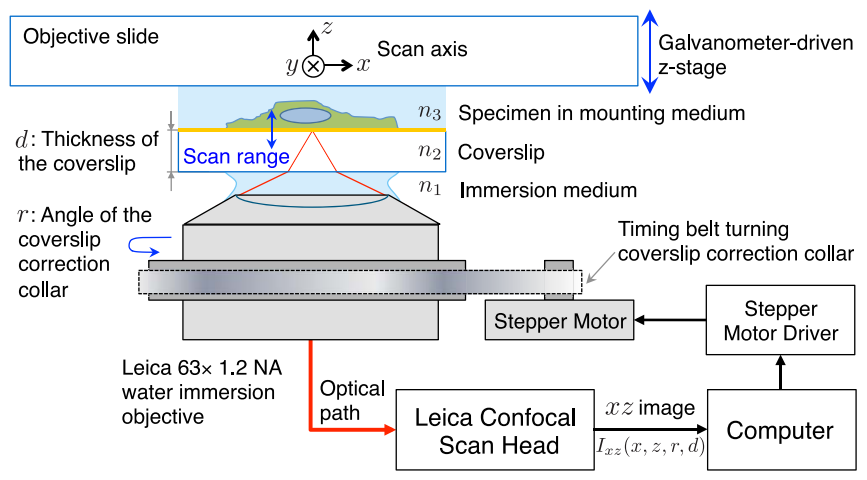

FIG. 2. A diagram of the automated adjustment system for coverslip thickness mismatch correction. $n_{2}$ is that of the coverslip. ${ }^{1,18-21}$ An axial scan model, based on the aberration model, ${ }^{19}$ is derived for a matched filter design (cf. Sec. IV).

The imaging intensity of a uniform reflective plane in confocal microscopy depends on the point spread function (PSF) and is given as follows: ${ }^{22}$

$$
I(x, y, z, f)=k^{-2}|\zeta|^{2} \Omega I_{\mathcal{L}} \operatorname{PSF}(2 \gamma z, f),
$$

where $x, y$, and $z$ are the coordinates at the imaged point as depicted in Fig. 1. $\operatorname{PSF}(z, f)$ denotes the one-dimensional point spread function along the $z$ axis through two media and $f$ denotes the actual focal depth in the second medium, which is the distance from the media interface to the actual focus position. $\gamma$ denotes the vertical direction ratio between the shifted actual focus position $f$, and the nominal focus position $f_{1}$ of the lens in medium 1, i.e., $f=\gamma f_{1}$, approximated to $\gamma=\frac{n_{2}}{n_{1}} \cdot{ }^{23}$ The term $\gamma z$ reflects the compression of the actual axial profile in the second medium ${ }^{24,25}$ due to the shift of the $z$ position by the galvanometer-driven $z$-stage as seen in Fig. 2. The scaling factor of 2 is obtained by the imaged focus movements against the reflective mirroric surface. The wave vector $k$, the reflectance $\zeta$ at the interface, the solid angle $\Omega$ of the aperture pupil as seen from either the object or image plane, and the illumination intensity $I_{\mathcal{L}}$ at pupil are assumed to be constant.

Applying the Debye approximation, ${ }^{19}$ the axial PSF with aberrations of a linearly polarized light source is obtained as follows:

$$
\operatorname{PSF}(z, f)=A^{2} I_{0}(z, f) \bar{I}_{0}(z, f),
$$

where $I_{0}$ denotes a diffraction integral (see Eq. (3)), and $\bar{I}_{0}$ denotes the complex conjugate of $I_{0}$. $A$ is a constant amplitude factor determined by the focal length of the lens in vacuo and the wavenumber of the first medium. The diffraction integral can be simplified as follows:

$$
I_{0}(z, f)=\int_{\cos \alpha}^{1} F_{0}(\beta) e^{i k_{0} \Psi(\beta, f, z)} d \beta,
$$

where

$$
\begin{gathered}
F_{0}(\beta)=2 \sqrt{\beta^{3}}\left(\frac{1}{\eta(\beta)+\beta}+\frac{\gamma^{-2} \eta(\beta)}{\beta+\gamma^{-2} \eta(\beta)}\right), \\
\Psi(\beta, f, z)=n_{1}\{f(\eta(\beta)-\beta)+z \eta(\beta)\}, \\
\eta(\beta)=\sqrt{\beta^{2}+\gamma^{2}-1},
\end{gathered}
$$

with $\beta=\cos \phi_{1}$, corresponding to the radial coordinate in the pupil plane (back focal plane). As shown in Fig. $1, \phi_{1}$ is the incident angle of the original focal point neglecting the refraction at the second medium. $\alpha$ is the maximum angle of $\phi_{1}$, which is determined by the numerical aperture (NA) of the objective lens and the refractive index of the immersion medium $n_{1} . \Psi(\beta, f, z)$ is called the aberration function, representing the wavefront along the radial coordinate $\beta$ at the axial position $z$ with the actual focal depth $f$ in the second medium. Here, the aberration function $\Psi$ represents spherical aberrations only, since $\Psi$ is solely dependent on the radial coordinate $\beta$. 
From Eq. (3), it can be seen that the actual focal depth through the second medium influences the amplitude of the aberration function $\Psi$. By adjustment of the angular position $r$ of the coverslip correction collar, it should compensate for this nominal focal depth at the reflective surface between coverslip and specimen. A linear relation between the adjustment $r$ and the thickness of the coverslip $d$ is assumed. ${ }^{11}$ Then, the effective actual focal depth for the spherical aberration can be rewritten as

$$
f=\underbrace{d-d_{0}}_{d_{m}}+b r+\gamma z
$$

where $b$ denotes the coefficient between the correction collar angle and the coverslip thickness mismatch. For the objective in Fig. $2 b=0.5 \mu \mathrm{m} /{ }^{\circ} . d_{m}$ is the residual mismatch at $r$ $=0$ and $z=0$, where the intensity along the $z$ axis is at its maximum. This can be obtained by $d$ with an initial coverslip mismatch correction at $r=0$, defined by $d_{0} \cdot \gamma z$ denotes the compressed focus movement due to vertical displacements.

Substituting Eq. (5) into Eq. (1), the $x z$ image, as recorded by the microscope, can be modeled as

$$
I_{x z}\left(x, z, r, d_{m}\right)=k^{-2}|\zeta|^{2} \Omega I_{\mathcal{L}} \operatorname{PSF}\left(2 \gamma z, d_{m}+b r+\gamma z\right),
$$

where $\operatorname{PSF}(\cdot, \cdot)$ is defined in Eqs. (2) and (3).

\section{B. Sample induced distortion in axial image measurements}

Considering the gain and offset of the detector, the axial imaging intensity of Eq. (6) can be rewritten as

$$
\hat{I}_{x z}\left(x, z, r, d_{m}\right)=K_{g}\left\{I_{x z}\left(x, z, r, d_{m}\right)+v(x, z)\right\}+K_{o f f}(r),
$$

where $K_{g}$ and $K_{\text {off }}$ denote a gain and offset of the detector and the term $v(x, z)$ represents the noise in the image, such as dark current and readout noise. ${ }^{26}$

Fig. 3 illustrates measured axial images of the coverslip specimen interface with (a) pure water as specimen, (b) a specimen of Convallaria majalis rhizome (CMR), ${ }^{17}$ and (c) a specimen of fixed mouse embryonic fibroblasts (MEFs, cf. Sec. V). Each reflection of specimen contains three sets of the coverslip correction collar setting, which are optimal

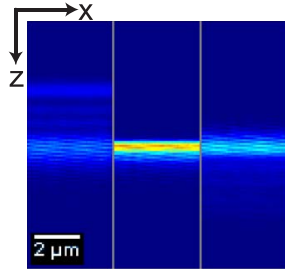

(a)

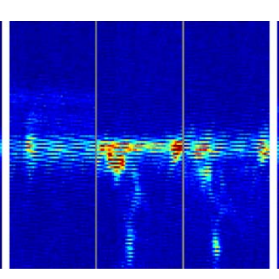

(b)

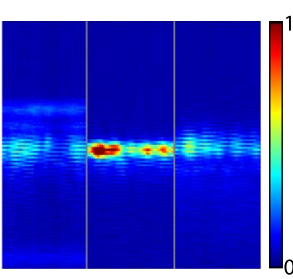

(c)
FIG. 3. Axial $x z$ images of the reflection of the coverslip interface with (a) pure water as specimen, (b) a specimen of Convallaria majalis rhizome (CMR), (c) a specimen of fixed mouse embryonic fibroblasts (MEFs). Each image consists of three interface images at the optimal correction (center), $-12.8 \mu \mathrm{m}$ (left), and $+12.8 \mu \mathrm{m}$ mismatch (right). The axial image is the brightest and sharpest at the optimal correction although the axial images with biological specimen fluctuated over $x$ axis due to the inhomogeneous optical structure of the specimen. correction (center), $-12.8 \mu \mathrm{m}$ (left), and $+12.8 \mu \mathrm{m}$ mismatch (right), corresponding to $r= \pm 25.2^{\circ}$. All images are made with the same laser power and detector gain setting and the pinhole at 1 airy unit. For all specimen, the reflection is the brightest at the optimal adjustment of the correction collar and blurred interference patterns are observed with a coverslip mismatch. Axial images with biological specimens, Figs. 3(b) and 3(c), show a large noise level along the $x$ axis, which is mainly caused by fluctuations in the refractive index of the specimen. The varying reflection due to the specimen, shown as bright irregular spots in Fig. 3, may cause erroneous mismatch estimates. In order to avoid signal saturation and low signal to noise ratio (SNR), which can cause an estimation error, measurement conditions such as laser intensity and detector gain need to be adjusted during the automated correction. To cope with these practical problems and to automate the spherical aberration correction, image processing steps are discussed in Sec. IV.

\section{AUTOMATED ADJUSTMENTS OF COVERSLIP THICKNESS MISMATCH}

The proposed automation of the coverslip mismatch correction should enable a fast and reliable correction with as low as possible photodamage to the specimen. Therefore, the number of axial scans that have to be recorded should be small only. For reliable correction, the correction accuracy should not depend on the kind of specimen and the measurement noise. Recording only a small number of image for obtaining the correction reduces the adjustment time while it also minimizes photodamage and photobleaching. ${ }^{27}$ In addition, the axial scans for correction should use low laser intensity to reduce the photodamage while low laser intensity leads to low SNR.

Fig. 4 illustrates the overall workflow for obtaining the best adjustment angle $r^{*}$ obtained from a set of noisy $x z$ images. A specified number of $x z$ images are recorded at different adjustment angles $r$. These images are further processed

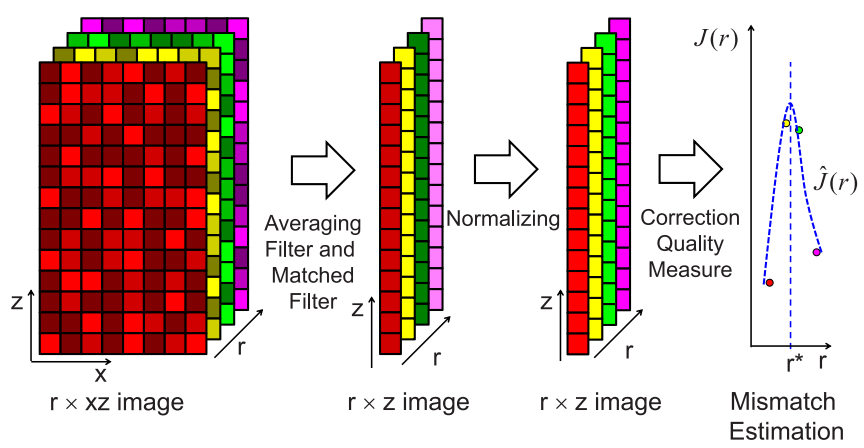

FIG. 4. Adjustment procedure of the proposed system. The small squares represent individual pixels of the image, the pixel colors (red, yellow, green, violet) indicate different adjustment angles $r$ of the correction collar and the shadowing of each pixel color represents the intensity fluctuation and noise. First, a specified number of $x z$ images are recorded at different adjustment angles $r$. Each $x z$ image is compressed into a $z$ image vector by averaging in $x$, followed by matched filtering. The $z$ image vectors are normalized to remove influences of measurement conditions. Finally, the $z$ image vectors are evaluated with quality functions and a fitting algorithm is applied to find the best adjustment angle $r^{*}$ 
by two filters. The first filter averages the images along the $x$ axis, reducing the $x$-position dependent intensity fluctuations. The second filter is a matched filter that reduces high frequency noise, which cannot be imaged by diffraction. Then a normalizer decreases the influence of the measurement conditions in order to provide comparable axial images. Finally, the correction quality of the filtered and normalized images are evaluated, and the optimal adjustment angle $r^{*}$ of the correction collar is obtained by a fitting algorithm.

\section{A. Noise reduction and image alignment}

To improve the SNR of the measured axial image, an averaging filter and a matched filter ${ }^{28}$ are applied with an offset rejection. The offset-corrected filtered image can be written as

$$
\begin{aligned}
I_{h}\left(z, r, d_{m}\right)= & \left(\frac{1}{2 x_{m}} \int_{-x_{m}}^{x_{m}} \hat{I}_{x z}\left(x, z, r, d_{m}\right) d x\right) * h_{m}(z) \\
& -\hat{K}_{o f f},
\end{aligned}
$$

where $x_{m}$ denotes the half of the averaging range of the $x$ axis, $*$ is the convolution operator, $h_{m}(z)$ is a matched filter, and $\hat{K}_{\text {off }}$ is the offset to be corrected for. The matched filter $h_{m}(z)$ is designed based on the axial image model without mismatch, i.e., $h_{m}(z)=\operatorname{PSF}(2 \gamma z, 0)$. This is because the spatial frequency response of axial images is bounded by the spatial frequency response of mismatch free images.

For every $x z$ image, averaging over $x$ axis is applied along the $x$ axis, generating a $z$ image vector per each adjustment $r$, reducing the $x$ dependency due to inhomogeneous specimen. Additionally, the averaging filter also reduces the noise term $v(x, z)$ of Eq. (7).

To compare the obtained $z$ image vectors, their maximum intensity position are aligned along the center $(z=0)$, and the edges of each axial image is removed symmetrically from a distance $z_{m}$. An offset rejector subtracts the background offset $\hat{K}_{\text {off }}$ of the detector, which is obtained by the mean intensity at either ends of the respective $z$ image. An $r z$ image is generated by stitching the $z$ image vectors along the collar angle $r$ to illustrate the evolution of the $z$ image along $r$ as Fig. 4. For the $r z$ image generation, $71 x z$ images that are equally spaced along the correction collar's full angular range are recorded and processed.

Fig. 5 illustrates the $r z$ images and corresponding correction quality measures (cf. Sec. IV C) without any noise filter (left), only with an averaging filter (center), and with averaging and matched filters (right image). For comparison, the correction measures are rescaled between 0 and 1 . Filtering smoothens the noisy $r z$ images and many local minima in the quality measures reduces. Therefore, this is advantageous to determine the optimal correction.

\section{B. Normalization of the axial image}

Saturation and low signal intensity may affect the evaluation of $z$ images during the measurement of the $x z$ images. At a lower signal intensity, i.e., the detection gain or the laser intensity are low, the detector noise dominates the evaluation of

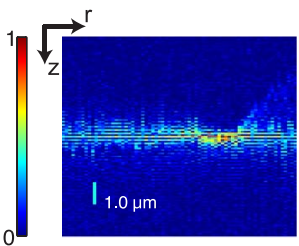

(a)
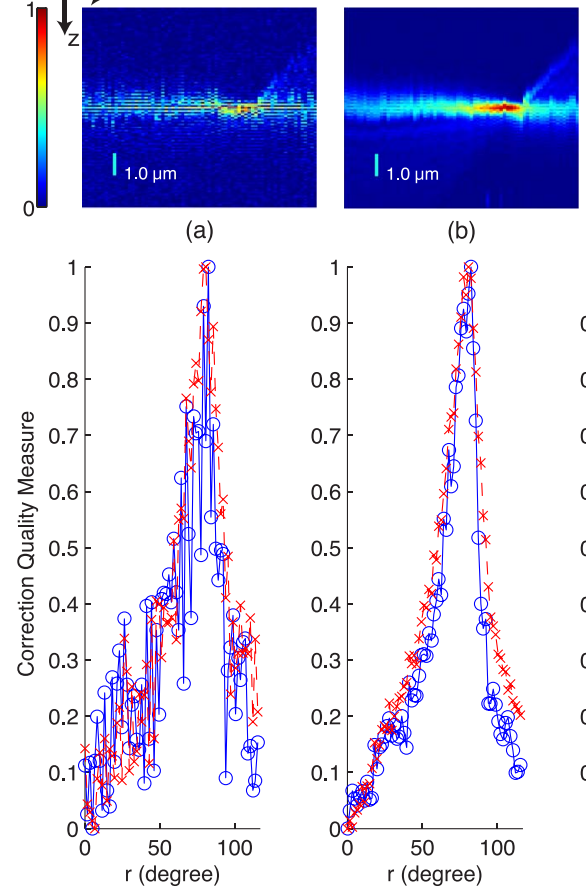

(b)

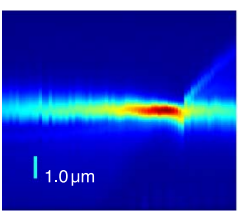

(c)
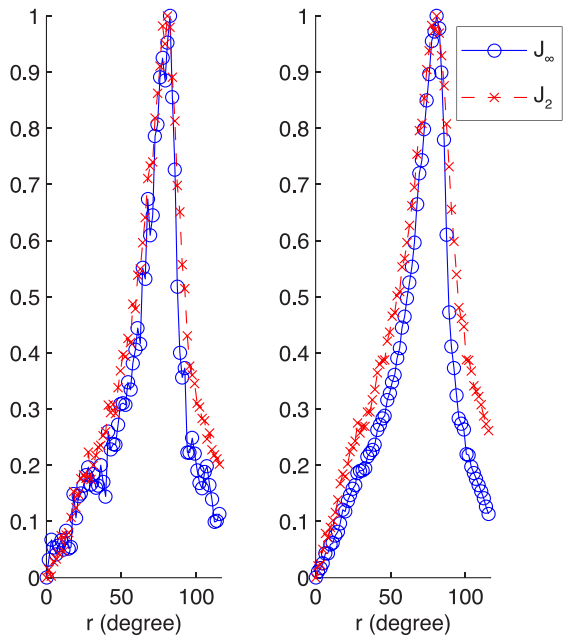

FIG. 5. $r z$ images and corresponding correction quality measures of the Leica standard sample of Convallaria majalis rhizome in case of (a) no filtering, (b) averaging filter only, (c) both averaging filter and matched filter. The averaging size is 50 pixels, which corresponds to $4 \mu \mathrm{m}$.

$z$ images. Detector saturation can occur during the correction due to the increased intensity by correction, which means loss of information at the peak of the image and results in wrong mismatch estimation as well. To solve this problem, a normalizer based on the integration of the axial image is applied as follows: ${ }^{29}$

$$
I_{n}\left(z, r, d_{m}\right)=\frac{I_{h}\left(z, r, d_{m}\right)}{\int_{-z_{m}}^{z_{m}} I_{h}\left(z, r, d_{m}\right) d z} .
$$

The integral in the denominator corresponds to the summation of the axial image, i.e., the magnitude of zero frequency of optical transfer function. ${ }^{22}$ With a normalizer, the detector gain can be freely adjusted to prevent a low signal as well as detector saturation. In addition, a normalizer also allows a comparison between measurements with specimen dependent intensity fluctuation.

Fig. 6 shows $r z$ images with a fixed detection gain (left) and with the detection gain adjustment (center) and with the detection gain adjustment after applying a normalizer (right). In Fig. 6(b), the gain of the detector is adjusted during the sweep of the correction collar $r$ in order to avoid detector saturation and low signal intensity. Abrupt changes of the maximum intensity along the $r$ axis depict the modification of the detector gain. Fig. 6(c) shows that a normalizer removes the dependency on the detection gain, resulting in a pattern similar to the image with the fixed detection gain in Fig. 6(a).

\section{Correction quality measures}

For the evaluation of the correction quality of the filtered and normalized axial images, three quality measures are 


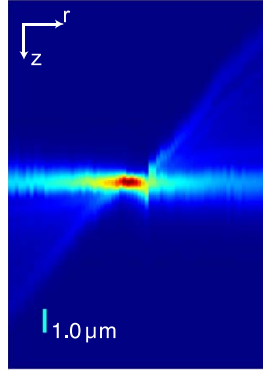

(a) (b)

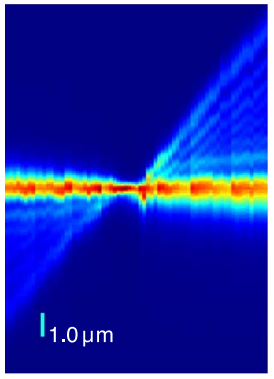

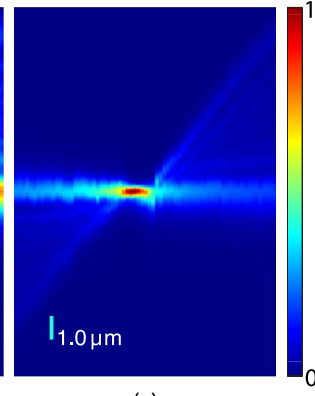

(c)
FIG. 6. $r z$ image of coverslip (a) fixed detection gain and (b) with a detection gain adjustment and (c) after applying the normalizer with the detection gain adjustment. The normalized $r z$ image (right) shows a similar diffraction pattern to the $r z$ image with the fixed detector gain (left). ${ }^{17}$ This allows mismatch estimation and correction regardless of detection gain and laser intensity.

defined, $\mathcal{L}_{\infty}$ and $\mathcal{L}_{2}$ norm, as well as entropy. A generalized quality measure, combining these three quality measures, is introduced.

$\mathcal{L}_{p}$ norms are proposed as a correction quality measure for a coverslip correction problem, ${ }^{16}$ and have been tested for $\mathcal{L}_{2}$. In this paper, we evaluate $\mathcal{L}_{2}$ as well as $\mathcal{L}_{\infty}$, which are given as

$$
\begin{gathered}
J_{\infty}\left(r, d_{m}\right)=\lim _{p \rightarrow \infty}\left(\int_{-z_{m}}^{+z_{m}} I_{n}^{p}\left(z, r, d_{m}\right) d z\right)^{1 / p}, \\
J_{2}\left(r, d_{m}\right)=\left(\int_{-z_{m}}^{+z_{m}} I_{n}^{2}\left(z, r, d_{m}\right) d z\right)^{1 / 2} .
\end{gathered}
$$

The maximum intensity $J_{\infty}$ corresponds to the Strehl ratio of the imaging system, which is defined as the ratio between the maximum intensity with and without aberrations. ${ }^{30} J_{2}$ is known as a measure for image sharpness. ${ }^{31,32}$

The entropy is proposed as a correction quality measure of the coverslip mismatch correction, which is defined as ${ }^{33,34}$

$$
J_{\text {ent }}\left(r, d_{m}\right)=-\int_{-z_{m}}^{+z_{m}} I_{n}\left(z, r, d_{m}\right) \ln I_{n}\left(z, r, d_{m}\right) d z .
$$

An entropy measure can only be used with a normalizer since normalized intensity can be interpreted as a probability density function of the reflection from the interface. Since entropy is known as information of the axial image, a smaller entropy correction measure means less uncertainty of the microscope image regarding the true object. $J_{\text {ent }}$ is a concave function with its minimum as optimum, in contrast to the $J_{\infty}$ and $J_{2}$ being optimal at the corresponding maximum.

In theory, the optimal correction position of the correction quality measures of $\mathcal{L}_{\infty}, \mathcal{L}_{2}$, and entropy should be the same without aberrations but in practice the optimum are different due to the intensity fluctuation by the specimen and the residual aberrations at the optimal correction. A generalized correction quality measure is proposed as a weighted linear combination of $\mathcal{L}_{\infty}, \mathcal{L}_{2}$, and inverse entropy measure, defined as

$$
J\left(r, d_{m}\right)=q_{1} J_{\infty}\left(r, d_{m}\right)+q_{2} J_{2}\left(r, d_{m}\right)+q_{3} J_{\text {ent }}^{-1}\left(r, d_{m}\right),
$$

where $q_{1}, q_{2}$, and $q_{3}$ are the weighting for each quality measure. Since each quality measure has different of its value, this weighting can be used for the equalization of the influence of each quality measure. It can be used to emphasize the important quality measure as well. Considering only the image sharpness $\mathcal{L}_{2}$, for example, the weights are chosen as $q_{2}=1$ and $q_{1}=q_{3}=0$.

\section{Mismatch estimation and optimal adjustment of correction collar}

It is desirable to find the optimal correction collar angle based on a low number of $x z$ images to be recorded. Three adjustment algorithms are discussed in detail and evaluated with specimen in Sec. V.

\section{Sweep method}

For the sweep method, $x z$ images are recorded at different correction collar positions $r$. This allows to extract a correction quality measure for each collar position and to determine the optimal adjustment by detecting the maximum quality measure. ${ }^{17}$

The sweep method is simple and easy to implement but it is time consuming as more $x z$ images have to be recorded. The precision is inversely proportional to the number of sweeping steps. For the above mentioned 71 measurements, the correction collar angle can be detected with a resolution of $1.65^{\circ}$, corresponding to $0.8 \mu \mathrm{m}$ coverslip thickness mismatch.

\section{Gaussian fitting method}

The GF method can be understood as an extension of the sweep method. Instead of increasing the accuracy by recording additional images, the optimal correction position is estimated by fitting a Gaussian function, given as ${ }^{16}$

$$
r^{*}=\underset{r}{\arg \min _{\hat{s}, r}} \sum_{t=0}^{m_{g}-1}\left\|J\left(r_{t}, d_{m}\right)-\left(\hat{s}_{1} e^{-\left(r_{t}-r\right)^{2} / \hat{s}_{3}^{2}}+\hat{s}_{2}\right)\right\|^{2},
$$

where $m_{g}$ is the number of axial images used, $\hat{s}=\left[\begin{array}{lll}\hat{s}_{1} & \hat{s}_{2} & \hat{s}_{2}\end{array}\right]$ is a parameter vector, and $r_{t}$ denotes the correction collar position defined by $r_{t}=t \Delta_{g}$ where $\Delta_{g}$ is the size of the sweeping step for Gaussian fitting. Gaussian fitting allows to interpolate between measurement points in order to reduce the number of axial images, however, it can also lead to a large estimation error when the Gaussian model does not match the real measured data.

\section{Coarse-fine Gaussian fitting (CF-GF) and mixed Gaussian fitting (MGF) method}

To minimize the model mismatch of the Gaussian model, an algorithm that is composed of two steps is proposed. In the first step, a coarse sweep of $m_{c} x z$ images with a sweeping step size of $\Delta_{c}$ is performed to estimate the coarse optimum $r_{c}^{*}$. In the second step, additional $x z$ images are recorded around the coarse optimum and the optimal adjustment is determined. The correction optimum $r^{*}$ is found by fine fitting 
of the nonlinear least squares problem

$$
r^{*}=\underset{r}{\arg \min _{\hat{\theta}, r}} \sum_{p=0}^{m_{f}-1}\left\|J\left(r_{p}, d_{m}\right)-\left(\hat{\theta}_{1} \hat{J}\left(r_{p}-r\right)+\hat{\theta}_{2}\right)\right\|^{2},
$$

where $\hat{J}$ denotes a model of the correction quality measure in (13) near the optimal correction under ideal measurement conditions. To this end measurements of coverslip with pure water as specimen have been recorded in advance in the range of $16.5^{\circ}$ near the optimal adjustments. The model $\hat{J}$ is generated based on the ideal images of the simulation of (1) and by applying the Curve Fitting Toolbox of Matlab to the measurement data. Two methods are defined by the choice of the fitting function, which are CF-GF method and CF-MGF method using Gaussian and mixed Gaussian function, respectively. $\hat{\theta}=\left[\hat{\theta}_{1} \hat{\theta}_{2}\right]$ is a parameter vector of a gain and offset of the model. $r_{p}$ denotes the correction collar position for fine fitting as

$$
r_{p}=r_{c}^{*}+\frac{2 p-m_{f}+1}{m_{f}-1} \Delta_{c} .
$$

$m_{f}$ is an odd number of $x z$ images used for the fine fitting, with $m_{f} \geq 5$. If $m_{f}=5$, for example, $\left\{r_{0}, r_{1}, r_{2}, r_{3}, r_{4}\right\}=\left\{r_{c}^{*}\right.$ $\left.-\Delta_{c}, r_{c}^{*}-0.5, r_{c}^{*}, r_{c}^{*}+0.5 \Delta_{c}, r_{c}^{*}+\Delta_{c}\right\}$ are used for the fine fitting. The total number of recorded images are $m_{c}+m_{f}$ -3 because 3 images of $r_{c}^{*}$ and $r_{c}^{*} \pm \Delta_{c}$ are already taken in the coarse sweep. With this two step algorithm significantly less images have to be recorded as compared to the sweep methods while the optimum can be detected at least with the same precision, which is experimentally validated in Sec. V.

\section{EXPERIMENT RESULTS}

\section{A. Evaluation of the automatic coverslip correction algorithms}

To evaluate the proposed mismatch correction methods, various samples are listed in Table I are examined. First, two coverslips with pure water as specimen in Sec. IV are examined as a reference. To demonstrate the robustness of the algorithms against specimen-induced intensity fluctuations, 5 coverslips with specimens are examined, gold particles on
poly-L-lysine (GP), CMR, fixed MEFs, and fixed human hepatocellular carcinoma cell line (Hep3B-AR). Particularity for Hep3B-AR, two slides are produced with different coverslips of standard thickness No. 1 (Hep3B-AR \#1) and No. 1.5 (Hep3B-AR \#1.5) for the comparison.

To obtain the optimal setting of the correction collar and to compare the adjustment methods, a set of $71 x z$ images at every $1.65^{\circ}$ of the correction collar $r$ are recorded. For the axial images, the $488 \mathrm{~nm}$ Ar laser is used as light source, except for the Hep3B-AR specimens, where the reflections of the $514 \mathrm{~nm}$ Ar laser are recorded since this wavelength is used for yellow fluorescent protein (YFP) excitation. The generalized correction quality measure Eq. (13) is used to evaluate the quality of correction with coefficients $q_{1}=2.8, q_{2}=4.2$, and $q_{3}=1$, which are chosen set all three correction quality measures equal.

Three correction methods in Sec. IV are evaluated with the following conditions. First, the entire set of $x z$ images processed directly by the sweep method for its optimum, which is set as reference. Second, 6 image subsets with 11 axial images are selected to evaluate the accuracy of the GF method. To simulate slightly different coverslip thicknesses and the corresponding shift in the $r z$ image, the subsets are equally spaced over $r$ with $m_{g}=11$ and $\Delta_{g}=9.9^{\circ}$. Finally in the CFGF and CF-MGF, 10 subsets of 6 axial images, $m_{c}=6$ and $\Delta_{c}=16.5^{\circ}$, are chosen for the coarse correction. The optimization of Eq. (15) is done with the correction measures of the $m_{f}=5$, i.e., with two additional axial images between the selected three images in the coarse correction. Therefore, the coarse-fine correction method uses in total $8 x z$ images, which significantly reduces the recording time as compared to the 71 images of the sweep method.

Table I shows the mean and standard deviation (STD) of the estimated optimal adjustments of each sample. The optimal adjustment obtained by the sweep method is considered as reference since it has a known accuracy of $\pm 1.65^{\circ}$. From the results, the specimen can be categorized into two groups, thin coverslips (Coverslip \#1, GP, CMR, MEFs, Hep3B-AR \#1) and thick coverslips (Coverslip \#1.5, Hep3B-AR \#1.5). GF method shows a large estimation error for thin coverslips and its standard deviation is always the largest except for Hep3B-AR \#1. The estimated optimal adjustment of CF-

TABLE I. Mean and standard deviation of the estimated optimal correction with sweep method, Gaussian fitting (GF) method, coarse-fine Gaussian fitting (CF-GF) method, and coarse-fine mixed Gaussian fitting (CF-MGF) method. Sweep method provides the reference measured optimum while the other methods estimate the optimal correction. Root mean square (RMS) of mean error and standard deviation describes the accuracy and the precision of the algorithms in

\begin{tabular}{|c|c|c|c|c|c|c|c|}
\hline \multirow[b]{2}{*}{ [deg] } & \multirow[b]{2}{*}{ Sweep } & \multicolumn{2}{|c|}{ GF } & \multicolumn{2}{|c|}{ CF-GF } & \multicolumn{2}{|c|}{ CF-MGF } \\
\hline & & Mean (error) & STD & Mean (error) & STD & Mean (error) & STD \\
\hline Coverslip \#1 & 77.55 & $69.42(-8.13)$ & 1.03 & $75.36(-2.19)$ & 0.68 & $77.45(-0.10)$ & 0.25 \\
\hline Coverslip \#1.5 & 52.8 & $51.46(-1.34)$ & 0.87 & $52.72(-0.08)$ & 0.69 & $54.57(1.77)$ & 0.25 \\
\hline GP & 77.55 & $74.3(-3.25)$ & 2.33 & $77.15(-0.4)$ & 0.61 & $78.67(1.12)$ & 0.28 \\
\hline CMR & 80.85 & $72.82(-8.03)$ & 4.07 & $78.94(-1.91)$ & 1.34 & $78.47(-2.38)$ & 0.83 \\
\hline MEFs & 89.10 & $101.21(12.11)$ & 15.52 & $89.05(-0.05)$ & 0.83 & $88.41(-0.69)$ & 0.98 \\
\hline Hep3B-AR \#1 & 74.25 & $68.64(-5.61)$ & 0.87 & $75.62(1.37)$ & 0.86 & $74.29(0.04)$ & 0.73 \\
\hline Hep3B-AR \#1.5 & 54.45 & 55.69 (1.24) & 1.92 & $55.38(0.93)$ & 0.71 & $54.24(-0.21)$ & 0.29 \\
\hline RMS performance & & $\ldots \quad(6.79)$ & 6.20 & $\ldots \quad(1.27)$ & 0.85 & $\ldots \quad(1.23)$ & 0.59 \\
\hline
\end{tabular}
different specimens. 
GF and CF-MGF shows a better accuracy with a small mean error, and the standard deviation of CF-MGF is smaller than CF-GF except for the case of MEFs. To evaluate the overall performance among the different specimen, root mean square (RMS) is used for the mean errors and the standard deviation. ${ }^{33}$ RMS of the mean error and RMS of the standard deviation represent the accuracy and the precision of the algorithm, respectively. It shows that the proposed coarse-fine correction methods (CF-GF, CF-MGF) provide the RMS mean error less than the sweep methods step, which is used as a reference and the precision with the smallest RMS mean error and standard deviation, only with $8 x z$ images while the sweep method and the GF method need 74 and 11 images.

\section{B. Imaging examples}

\section{Evaluating the PSF}

In order to determine the PSF, $20 \mathrm{~nm}$ gold particles (Gold Colloid, BBInternational, Cardiff, UK), which are sparsely distributed on a Poly-L-Lysine coated coverslip are used as the first specimen, because the refection of particles smaller than the wavelength of the laser shows a squared PSF with spherical aberrations. $^{22}$ In addition, gold particles do not bleach so that reflection intensity does not degrade by the former measurement trials, i.e., aberrations are the only factor that degrades the image. An Ar laser with a wavelength of $488 \mathrm{~nm}$ is used for imaging.

Fig. 7 shows axial images of a gold particle recorded with the confocal microscope (c) with the optimal adjustment of the correction collar and (a), (b), (d), (e) with a mismatch of $\pm 26.5^{\circ}$ in the correction collar angle, corresponding to a thickness mismatch of $\mp 13.2 \mu \mathrm{m}$. The gain is fixed in (b)-(d) to show the low intensity of the PSF in (b) and (d) due to spherical aberrations. (a) and (e) are recorded at the same condition of the correction collar as in (b) and (d) but with the detector gain adjusted to obtain brighter images, clearly illustrating the enlarged shape of the PSF. This is based on a scenario that users frequently do: not adjusting the correction collar but the detector gain in order to obtain the confocal microscope image with enough brightness. These gain adjusted PSFs are close to the images of unexperienced microscope users who usually adjust the detector gain or the laser intensity to make the image bright rather than manually adjusting

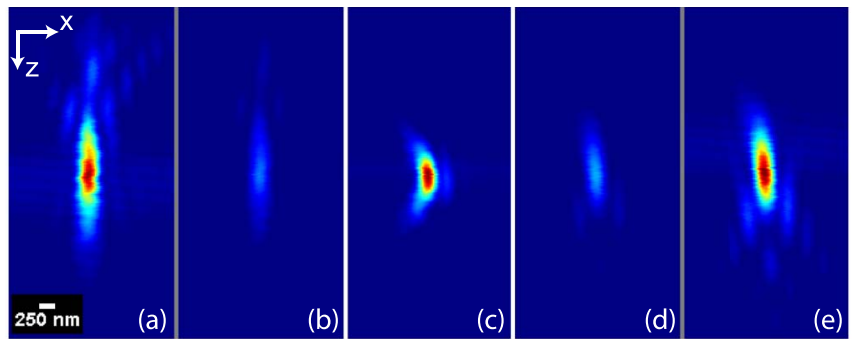

FIG. 7. $x z$ reflection images of a $20 \mathrm{~nm}$ gold particle with (c) the optimal adjustment, (a) and (b) $-13.2 \mu \mathrm{m}$ mismatch, and (d) and (e) $+13.2 \mu \mathrm{m}$ mismatch, corresponding to $\pm 26.4^{\circ}$ of the correction collar angle. The detector gain is fixed in (b)-(d), while (a) and (e) are recorded with an increased detector gain to achieve the same maximum intensity as in (c). The difference in the PSF size is clearly visible among (a), (c), and (e).
TABLE II. Relative maximum intensity with respect to the optimal adjustments and lateral and axial full width at half maximum (FWHM) of the measured point spread function.

\begin{tabular}{lccc}
\hline \hline & Optimum & $13.2 \mu \mathrm{m}$ & $-13.2 \mu \mathrm{m}$ \\
\hline Relative maximum intensity & 1 & 0.30 & 0.24 \\
Lateral FWHM $[\mu \mathrm{m}]$ & 0.20 & 0.24 & 0.22 \\
Axial FWHM $[\mu \mathrm{m}]$ & 0.52 & 0.78 & 0.94 \\
\hline \hline
\end{tabular}

the correction collar. Table II summarizes relative maximum intensity as compared to the optimal adjustments, as well as the lateral and axial full width at half maximum (FWHM) of the measured particle images. It is observed that in the uncompensated case the maximum intensity drops by up to $76 \%$ and the axial FWHM also increases significantly by up to $80 \%$.

\section{Automated optiamal adjustment correction collar in multicolor images}

To demonstrate the improved image quality for the optimally adjusted correction collar, fixed MEFs are used as the second specimen. The cytoskeleton of MEFs is visualized by means of immunofluorescent labeling of beta-tubulin (Alexa488) and with a chromatin staining (DAPI) ${ }^{35}$ that are excited by a $488 \mathrm{~nm}$ Ar laser and a $405 \mathrm{~nm}$ diode laser and recorded by each PMT simultaneously. Beta-tubulin as a dimer with alpha-tubulin assemble in cells into a hollow cylindrical structure of approximately $24 \mathrm{~nm}$ diameter, the microtubules. ${ }^{36}$ This is smaller than the diffraction limit of the confocal microscope and can be a useful indicator with a high sensitivity to aberrations.

For a comparison of fluorescence images of MEFs between a spherically aberrated case and the optimally adjusted microscope, the optimal adjustment of Hep3B-AR \#1.5 is chosen as the unadjusted case for. This scenario is likely when multiple users share the same microscope and the objective lens but use different coverslips for their experiments. Fig. 8 shows $r z$ images and the corresponding generalized correction quality of Hep3B-AR \#1.5 and MEFs. The optimal adjustments of Hep3B-AR \#1.5 and MEFs, $r_{H}^{*}$ and $r_{M}^{*}$, differ by $34.7^{\circ}$ as shown in $r z$ images (a) and (b), corresponding to $17.3 \mu \mathrm{m}$ thickness mismatch. 3D images of MEFs for the uncompensated and compensated case are taken to have the same voxel height $z$ as width in $x$ and $y$. The 3D images of the compensated and uncompensated cases are aligned based on the maximization of the cross-correlation between two 3D images, in order to locate the same position as well as to remove the effect of defocus by a different $z$ position. Microtubule images are used for the alignment of images, because of their complex pattern.

Fig. 9 shows lateral $x y$ fluorescence images and axial $x z$ fluorescence images of chromatin (DAPI, Cyan) and betatubulin (Alexa488, Green) for (a) the uncompensated case and (b) the optimally adjusted correction collar. In the uncompensated case, the image intensity of both chromatin and betatubulin clearly degrades in the lateral image. In the $x z$ image, in addition, the image of both fluorophores are elongated and dispersed. Furthermore, a misalignment in the axial position 

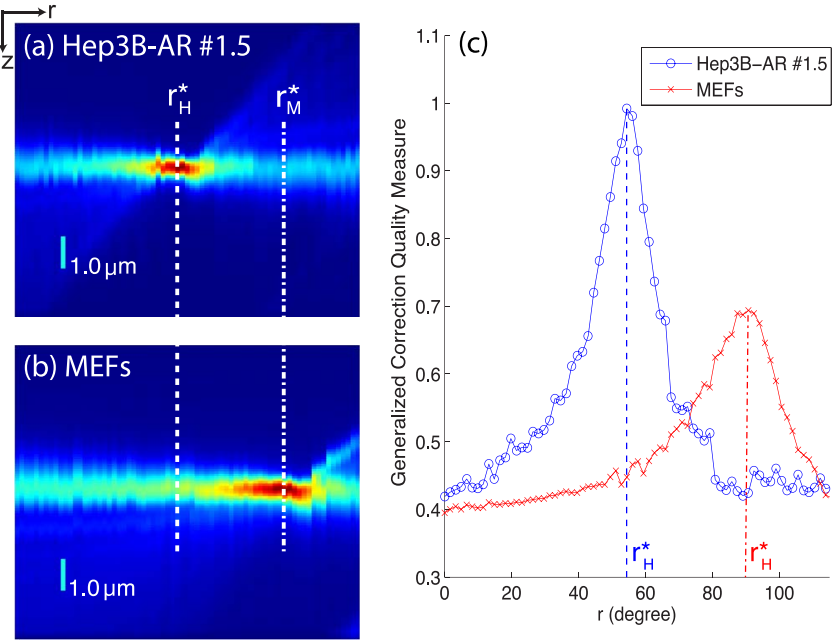

FIG. 8. Measured $r z$ image of a slide of (a) a fixed human hepatocellular carcinoma cell line with a coverslip of standard thickness No. 1.5 (Hep3B-AR $\# 1.5$ ) and (b) a fixed mouse embryonic fibroblasts (MEFs). The optimal adjustment of Hep3B-AR \#1.5, $r_{H}^{*}$ (dashed line), is different from that of MEFs, $r_{M}^{*}$ (dashed dotted line), indicating the necessity of individual adjustment of the correction collar before imaging each sample. (c) The generalized correction quality clearly shows the different maxima in correction quality along $r$ axis.

between the images of each color can be observed in the uncompensated case. This denotes that the fluorescence images of different excitation wavelengths have a shifted axial focus when a spherical aberration due to the coverslip thickness mismatch exists. In case of the optimally adjusted correction collar (b), the image intensity, sharpness, as well as the alignment is improved, which is clearly visible in the lateral as well as axial images.

\section{Optimal adjustment of correction collar vs adjustment of detection gain}

To demonstrate the improvement in image quality by optimally adjusting the correction collar as compared to

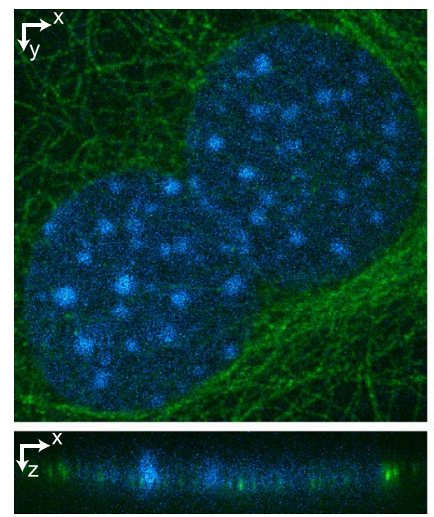

(a)

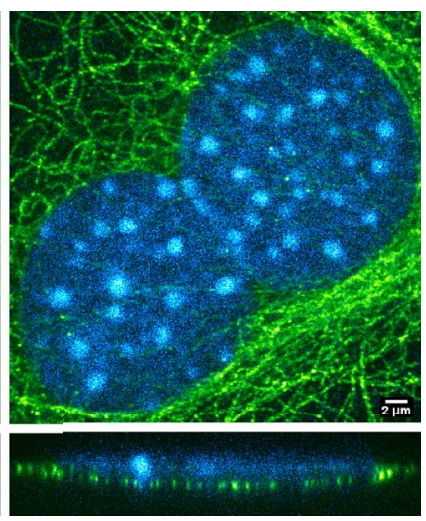

(b)
FIG. 9. Fluorescence images of chromatin (DAPI, Cyan) and betatubulin (Alexa488, Green) in fixed mouse embryonic fibroblasts (MEFs) with (a) uncompensated spherical aberrations $\left(r_{H}^{*}\right)$ and (b) optimally compensated $r_{M}^{*}$. The image degradation is significant in axial $x z$ images, and the axial location of the chromatic cluster is aligned with the beta-tubulin image in (b) while it is not aligned in (a). (Multimedia view) [URL: http://dx.doi.org/10.1063/1.4904370.1] [URL: http://dx.doi.org/10.1063/1.4904370.2] enhancing the detection gain (cf. Fig. 7) in a real imaging example, a fixed human hepatocellular carcinoma cell line (Hep3B-AR) is imaged with the confocal microscope. Hep3B-AR is grown on coverslips with the standard thickness No. 1.5 (sample Hep3B-AR \#1.5), stably expressing the androgen receptor (AR), double labeled with YFP at the $\mathrm{N}$-terminus and cyan fluorescent protein (CFP) at the Cterminus of the protein. ${ }^{37}$ The AR is a hormone activated transcription factor that regulates the expression of genes involved in the development and maintenance of the male phenotype as well as prostate cancer growth. The hormone (R1881) activated AR shows a typical sprinkled distribution which is correlated with transient binding to DNA and is linked to the spatial distribution of transcriptional activity. ${ }^{38}$ An Ar laser with a wavelength of $514 \mathrm{~nm}$ is used to excite YFP.

3D images of YFP-labeled AR of Hep3B-AR \#1.5 are recorded with the uncompensated system and the optimally adjusted correction collar, respectively, by stacking $320 x z$ image layers to obtain the same $y$ voxel width as for the $x z$ voxel width and height and aligned by the maximization of the cross-correlation. In the uncompensated case, the images are recorded with two detector gains, which is once the same gain as in the compensated case and once with an adjusted gain for similar brightness as in the compensated case. The uncompensated image with an adjusted detector gain is quite likely in practical imaging application (cf. Fig. 7).

Fig. 10 shows lateral $x y$ images and corresponding sectional $x z$ image slices of the fluorescently labeled AR (Yellow) in Hep3B-AR cells (coverslip \#1.5) with (a) the uncompensated case, (b) the uncompensated case with adjusted detector gain, and (c) the optimally adjusted correction collar. The image degradation is more significant in the axial images due to the shape of the PSF (cf. Fig. 7). The elongated and dispersed features are observed, making their localization difficult while the sample details in the uncompensated images are blurred and distorted.

In summary, the automated and optimal adjustment of the correction collar proposed here minimizes the spherical aberrations and enables sharp and bright images with the scanning confocal microscope.

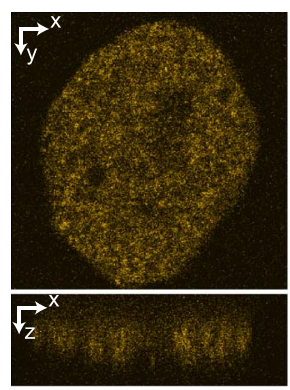

(a)

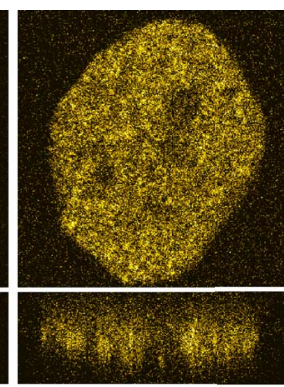

(b)

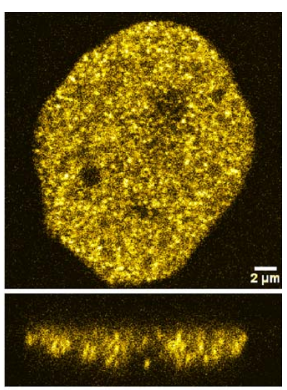

(c)
FIG. 10. Images of fluorescently labeled androgen receptor (YFP, yellow) in the nucleus of a fixed human Hep3B-AR cell. Spherical aberrations are (a) uncompensated $\left(r_{M}^{*}\right)$, (b) also uncompensated with an adjusted detector gain, and (c) optimally compensated $\left(r_{H}^{*}\right)$. Details of fluorescent clusters are blurred in the uncompensated images and cannot be enhanced by increasing the detector gain. (Multimedia view) [URL: http://dx.doi.org/10.1063/1.4904370.3] [URL: http://dx.doi.org/10.1063/1.4904370.4] 


\section{CONCLUSION}

This paper presents the automatic adjustment of spherical aberration correction with a motorized correction collar. The proposed approach is evaluated for compensation of spherical aberrations due to coverslip mismatch. After noise filtering and normalization, the measured axial images are evaluated by general correction quality measures, including the maximum intensity, image sharpness, and image entropy. For searching the optimal correction, the sweep method, Gaussian fitting method, and coarse-fine Gaussian fitting method are discussed. These algorithms are evaluated with 7 specimens and it is shown that the coarse-fine Gaussian correction has the best adjustment accuracy with least number of images to be recorded, which minimize the adjustment time as well as bleaching of the sample. In a practical lab scenario is shown that the proposed automated adjustment minimizes spherical aberrations, resulting in the smallest PSF, the highest intensity, and the best alignment of multi-color images. This enables recording of the sharpest lateral as well as axial resolution with a minimized distortion of the confocal microscope images.

Ongoing research is focused on the integration of wavefront sensing and compensation of the aberrations in general including non-spherical, by means of adaptive optics, which enhances the image quality even further.

\section{ACKNOWLEDGMENTS}

This research has been supported by the Integrated Smart Microscopy project in the Smart Optics Systems program of Technologiestichting STW, the Netherlands. The authors would like to thank Jeffrey van Haren from Erasmus MC Rotterdam, for providing MEFs specimen slides, and Rene Paris, Markus Thier, and Francesco Cigarini from ACIN at Vienna University of Technology for the fruitful discussion. The authors would like to thank Tanjef Szellas, Andrea Pfeiffer, and Henk den Hartog from Leica Microsystems for providing the confocal microscope and technical support.

\footnotetext{
${ }^{1}$ Handbook of Biological Confocal Microscopy, 3rd ed., edited by J. B. Pawley (Springer Science Business Media, 2006).

${ }^{2}$ D. Ehrhardt, Curr. Opin. Plant Biol. 6, 622 (2003).

${ }^{3}$ B. N. Giepmans, S. R. Adams, M. H. Ellisman, and R. Y. Tsien, Science 312, 217 (2006).

${ }^{4}$ R. M. Hoffman, Nat. Rev. Cancer 5, 796 (2005).

${ }^{5}$ M. R. Fein and M. Egeblad, Dis. Model. Mech. 6, 580 (2013).
}

${ }^{6} \mathrm{~L}$. Bakota and R. Brandt, International Review of Cell and Molecular Biology (Academic Press, 2009), Vol. 276, pp. 49-103.

${ }^{7}$ S. B. Andersson, Appl. Phys. B 80, 809 (2005).

${ }^{8}$ Glycerol Objective (Leica Microsystems, 2004).

${ }^{9}$ M. J. Booth and T. Wilson, J. Microsc. 200, 68 (2000).

${ }^{10}$ M. Shaw, S. Hall, S. Knox, R. Stevens, and C. Paterson, Opt. Express 18, 6900 (2010).

${ }^{11}$ R. Liang, Optical Design for Biomedical Imaging (SPIE Press, 2010).

${ }^{12}$ M. Shimada, Y. Shimada, and H. Kitagawa, U.S. patent 6,563,634 (13 May 2003).

${ }^{13}$ M. Karasawa, A. Yoshikawa, and K. Hayashi, U.S. patent 7,825,360 (2 November 2010).

${ }^{14}$ W. Knebel, T. Bauer, and P. Euteneuer, U.S. patent 8,724,103 (13 May 2014)

${ }^{15}$ C. Monks, Imaging Microsc. 3, 48 (2004), available online at http://www.imaging-git.com/magazine/imaging-microscopy-3-2004.

${ }^{16}$ M. Schwertner, M. J. Booth, and T. Wilson, J. Microsc. 217, 184 (2005).

${ }^{17}$ H. W. Yoo, M. Verhaegen, M. E. van Royen, and G. Schitter, in Proceedings of the IEEE International Instrumentation and Measurement Technology Conference (I2MTC) (IEEE, 2012), pp. 1083-1088.

${ }^{18}$ S. Hell, G. Reiner, C. Cremer, and E. H. K. Stelzer, J. Microsc. 169, 391 (1993).

${ }^{19}$ P. Török, P. Varga, Z. Laczik, and G. R. Booker, J. Opt. Soc. Am. A 12, 325 (1995).

${ }^{20}$ M. J. Booth, M. A. A. Neil, and T. Wilson, J. Microsc. 192, 90 (1998).

${ }^{21}$ M. J. Booth and T. Wilson, J. Biomed. Opt. 6, 266 (2001).

${ }^{22} \mathrm{~J}$. Mertz, Introduction to Optical Microscopy, 1st ed. (Roberts and Company Publisher, 2009).

${ }^{23}$ K. Carlsson, J. Microsc. 163, 167 (1991).

${ }^{24}$ W.-L. Chen, Y.-Y. Lee, C.-Y. Chang, H.-M. Huang, T.-C. Lu, and Y.-M. Chang, Rev. Sci. Instrum. 84, 113108 (2013).

${ }^{25}$ K. J. Baldwin and D. N. Batchelder, Appl. Spectrosc. 55, 517 (2001).

${ }^{26} \mathrm{Q}$. Wu, F. Merchant, and K. Castleman, Microscope Image Processing (Academic Press, 2008).

${ }^{27}$ D. Debarre, E. J. Botcherby, T. Watanabe, S. Srinivas, M. J. Booth, and T. Wilson, Opt. Lett. 34, 2495 (2009).

${ }^{28}$ H. L. V. Trees, Detection, Estimation, and Modulation Theory, Part I (Wiley-Interscience, 2001).

${ }^{29}$ M. Bravo-Zanoguera, B. von Massenbach, A. L. Kellner, and J. H. Price, Rev. Sci. Instrum. 69, 3966 (1998).

${ }^{30}$ Adaptive Optics for Astronomical Telescopes, edited by J. W. Hardy (Oxford University Press, USA, 1998).

${ }^{31}$ A. Buffington, F. S. Crawford, R. A. Muller, A. J. Schwemin, and R. G. Smits, J. Opt. Soc. Am. 67, 298 (1977).

${ }^{32}$ L. P. Murray, J. C. Dainty, and E. Daly, Proc. SPIE 5823, 40 (2005).

${ }^{33}$ Y. Sun, S. Duthaler, and B. J. Nelson, Microsc. Res. Tech. 65, 139 (2004).

${ }^{34}$ R. K. Tyson, Principles of Adaptive Optics, 3rd ed. (CRC Press, 2011).

${ }^{35}$ K. Drabek, M. van Ham, T. Stepanova, K. Draegestein, R. van Horssen, C. L. Sayas, A. Akhmanova, T. ten Hagen, R. Smits, R. Fodde, F. Grosveld, and N. Galjart, Curr. Biol. 16, 2259 (2006).

${ }^{36}$ C. Conde and A. Cáceres, Nat. Rev. Neurosci. 10, 319 (2009).

${ }^{37}$ M. E. van Royen, W. A. van Cappellen, C. de Vos, A. B. Houtsmuller, and J. Trapman, J. Cell Sci. 125, 1970 (2012).

${ }^{38}$ M. E. van Royen, S. M. Cunha, M. C. Brink, K. A. Mattern, A. L. Nigg, H. J. Dubbink, P. J. Verschure, J. Trapman, and A. B. Houtsmuller, J. Cell Biol. 177, 63 (2007). 